\title{
Masseter Muscle Activity in Orthodontically Treated Patients with a History of \\ Temporomandibular Joint Disorder: \\ An Electromyographic Study
}

Submitted: $21 / 01 / 2021$

Accepted: 28/09/2021

\author{
Nisallina Apridini, Nia Ayu Ismaniati Noerhadi*, Erwin Siregar \\ Department of Orthodontics, Faculty of Dentistry, Universitas Indonesia, \\ Fakarta 10430, Indonesia
}

*Corresponding author: nia.noerhadi@gmail.com

To cite this article: Apridini N, Noerhadi NAI, Siregar E (2021). Masseter muscle activity in orthodontically treated patients with a history of temporomandibular joint disorder: An electromyographic study. Arch Orofac Sci, 16(2): 191-198. https://doi.org/10.21315/aos2021.16.2.9

To link to this article: https://doi.org/10.21315/aos2021.16.2.9

\begin{abstract}
The present study aimed to evaluate the surface electromyography (sEMG) activity of the masseter muscles in patients with a history of temporomandibular joint disorder (TMJD) who received orthodontic treatment. In total, 22 participants aged 18-35 years old were included in this study. They were divided into the control group (patients without a history of TMJD $[n=11]$ ) and the test group (those with a history of TMJD $[n=11]$ ). Each participant underwent sEMG of the right and left masseter muscles at 5-s maximum voluntary contraction (MVC). Results showed that the TMJD group had a lower sEMG activity of masseter muscles at MVC than the non-TMJD group. However, the differences were not statistically significant $(p>0.05, t$-test). The Spearman's correlation coefficient test revealed a weak negative correlation between muscle activity on sEMG and history of TMJD $(p>0.05)$. In conclusion, orthodontically treated patients in TMJD group have reduced masseter muscle activity during MVC, compare to the non-TMJD group.
\end{abstract}

Keywords: Orthodontic treatment, temporomandibular joint disorder, masseter muscles, electromyography

\section{INTRODUCTION}

Temporomandibular joint disorder (TMJD) is a relatively common condition affecting the physiological functions of the stomatognathic system. TMJD is frequently associated with problems in the masticatory system, which is composed of the masticatory muscles, temporomandibular joint (TMJ), and surrounding structures. The characteristics of TMJD include TMJ and/or masticatory muscle pain, TMJ sounds, and chewing difficulties. Moreover, TMJD is correlated with trauma, anatomical structure, also pathophysiological and psychosocial factors. Malocclusion and occlusal interference are also considered to be the risk factors of TMJD (Henrikson \& Nilner, 2003).

Clinical examinations and radiographic imaging, such as magnetic resonance imaging and computed tomography scan are the gold standard procedures for diagnosing TMJD (Tartaglia et al., 2011; Politti et al., 2016). Whilst functional examination of stomatognathic system to measure masticatory muscle activity could be evaluated by using the electromyography 
(EMG) (Botelho et al., 2009; Al-Saleh et al., 2012; Masci et al., 2013). EMG is used to evaluate muscle activity by detecting the electrical signals generated during muscle contraction (Al-Saleh et al., 2012; Masci et al., 2013; Sumonsiri \& Thongudomporn, 2017).

Surface (sEMG) is a noninvasive method in which electrodes are placed on the skin surface overlying the muscles. It is more commonly used than the needle EMG method (Al-Saleh et al., 2012). In the field of clinical orthodontics, EMG has been used to assess the effect of occlusion on neuromuscular balance in the stomatognathic system and the functional outcome of orthodontic treatment (Masci et al., 2013). After orthodontic treatment, patients with asymmetric malocclusion can present with normal masticatory muscle activities on EMG. This result can be attributed to the achievement of occlusal balance, which could regain neuromuscular balance (Botelho et al., 2009). It had been reported that in patients with TMJD, the complaints were muscle pain upon palpation and mandibular movement reduced after the orthodontic treatment (Henrikson \& Nilner, 2003).

The masticatory muscles comprise four different muscles, which are as follows: the masseter, anterior temporal, medial pterygoid and lateral pterygoid muscles. Among the four masticatory muscles, the masseter muscle play an important role in the stomatognathic system, so it was commonly investigated in previous studies. Anatomically, this muscle is located superficially under the skin surface, so that it can be easily palpated and examined on sEMG. Thus, the present study aimed to evaluate the masseter muscles activity in patients with a history of TMJD who had received orthodontic treatment. Moreover, the correlation between history of TMJD and masseter muscle activity on EMG after orthodontic treatment was also evaluated.

\section{MATERIALS AND METHODS}

The ethics committee of Universitas Indonesia approved the present study (No: 106/Ethical Approval/FKG UI/ IX/2019). Patients who had completed the orthodontic treatment in the Orthodontic Clinic at Dental Hospital, Faculty of Dentistry, Universitas Indonesia, at least two years prior to the study were recruited by convenience sampling. Sample size was calculated using sample size formula for two independent sample. In total, 22 patients ( 5 men and 17 women) had participated in this analysis and they were divided into two groups. Group 1 consisted of 11 participants without a history of TMJD (control group). On the other hand, Group 2 consisted of 11 participants with a history of TMJD (test group). Informed consent was obtained. The inclusion criteria were as follows: male or female patients (aged 18-37 years old) with or without a history of TMJD who completed their orthodontic treatment. The patients had good molar relationship in the sagittal, vertical and transversal dimensions. Molar and canine had Class I or Class II half unit relationship with mild crowding, normal overjet and normal overbite (1-4 $\mathrm{mm}$ ). Meanwhile, the exclusion criteria were patients with parafunctional habits, facial asymmetry, history of neuromuscular disease, or trigeminal and facial nerve disorders.

\section{sEMG Examination}

The examination of muscle activity was performed using Cadwell EMG Sierra ${ }^{\circledR}$ Summit in the Neurology Clinic, Cipto Mangunkusumo Hospital, Jakarta, Indonesia. Each participant simultaneously underwent sEMG examination of the left and right masseter muscles. Before the examination, the participants were required to sit in an upright position. The Frankfurt horizontal plane and inter-pupillary line were parallel to the floor. Then, they were instructed to assume the same position until the examination was completed. The skin surface on which the electrodes were placed was initially prepared with an alcohol swab. 
The electrodes were put on the muscle belly (the bulging point during tooth clenching). Two electrodes with a $2-\mathrm{cm}$ distance were placed on the left and right side of the masseter muscles. Data on muscle activity during isometric muscle contraction were obtained by asking the participant to bite the cotton rolls (maximum voluntary contraction [MVC]) bilaterally for 5 sec. Fig. 1 shows the raw signal of the muscle activity recorded during sEMG examination.

The examinations were performed by a researcher who was supervised by a neurologist. To assess the external reliability, the participants were tested twice by both researcher and neurologist. Ensuring the internal reliability, the examination conducted by the researcher was repeated twice for each participant with an interval of 15 min between measurements. To minimise bias, the researcher was blinded to the control and test groups of each participant during data analysis.

\section{EMG Data Processing}

Output data from the muscle activity examination resulted in ASCII data, which comprised of the raw data of the signal amplitudes during the $5 \mathrm{sec}$ recording.
The ASCII data obtained were then used to calculate the root mean square (RMS) amplitude values in microvolt $(\mu \mathrm{V})$. ASCII data processing and calculation of RMS amplitude values were conducted using Microsoft Excel.

\section{Statistical Data Analysis}

Univariate analysis was performed to assess the frequency distribution of data on mean, maximum, minimum and standard deviation for the RMS amplitude in each group. The data reliability tests were performed for both intra- and inter-examiners using the intraclass correlation coefficient (ICC) test. The coefficient values showed that the intraexaminer and inter-examiner reliability were excellent (ICC > 0.8). The Shapiro-Wilk test revealed that the data had a normal distribution and the data homogeneity was quite good. Thus, an independent $t$-test was conducted to compare the masseter muscle activity between the control and study groups. Then, the Spearman's correlation test was performed to assess the association between history of TMJD and masseter muscle activity. A $p$ value $<0.05$ was considered statistically significant. Statistical analyses were performed using software IBM SPSS Statistics version 20.

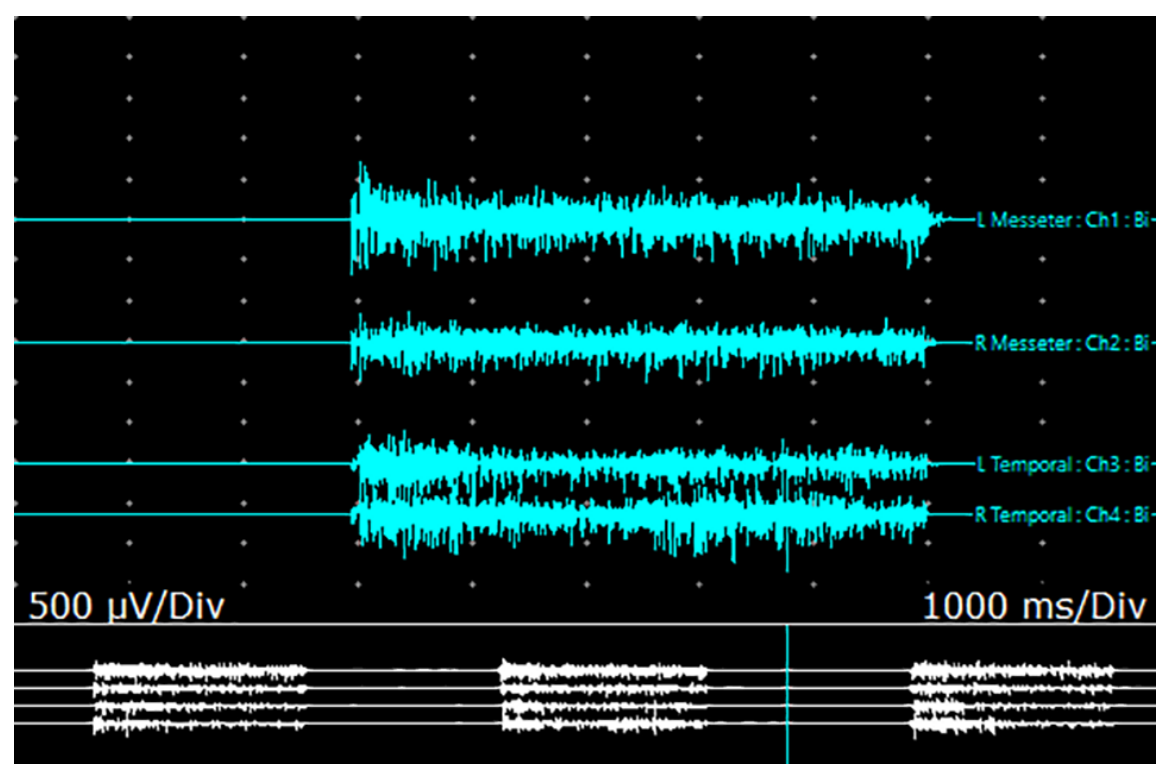

Fig. 1 EMG: Signal waves of the masticatory muscle activity at MVC. 


\section{RESULTS}

Participants in the present study consisted of $23 \%$ males $(n=5)$ and $77 \%$ females $(n=17)$. The distribution of participants between control and test group based on gender did not significantly differ $(p>0.05)$. Meanwhile, a significant difference was found between groups in terms of age. The mean age for the control group and the test group were $24.09 \pm 3.24$ years and $30.91 \pm 3.39$ years, respectively $(p>0.05)$ (Table 1).

\section{EMG Activity of the Masseter Muscles}

The sEMG activity examination revealed that there was a difference of RMS amplitude values between the control group and the test group as shown in Table 2. Although the differences between the two groups were not statistically significant $(p>0.05)$, the control group was tend to have a higher RMS amplitude value compared to the test group. On the left masseter muscle, higher RMS amplitude values $(p>0.05)$ were found in the control group (113.88 \pm 53.44$)$ compared to the test group $(105.39 \pm 48.40)$. Similar results were found on the right side of masseter muscle, RMS amplitude values in the control group $(107.92 \pm 48.06)$ were higher than in the test group $(102.75 \pm 62.76)$ with $p$ value $>0.05$.

\section{EMG Activity and History of TMJD}

The Spearman's correlation test revealed a weak negative correlation between history of TMJD and EMG activity of the masseter muscles. The $r_{s}$ value of left masseter muscles was -0.093 and right masseter muscle was -0.150 , with no significant correlation was observed $(p>0.05)$ as shown in Table 3.

Table 1 Distribution of participants based on gender and age

$\begin{array}{lccccc}\text { Sex } & \text { Age }(\text { mean } \pm \text { SD }) & \text { Non-TMJD group } & \text { TMJD group } & \text { Total } & p \\ \text { Male } & 27.6 \pm 4.78 & 2 & 3 & 5 & 17 \\ \text { Female } & 27.47 \pm 4.90 & 9 & 8 & 22 & 0.00 \\ \text { Total } & 27.50 \pm 4.76 & 11 & 11 & 30.91 \pm 3.39 & \\ \text { Age (mean } \pm \text { SD) } & & 24.09 \pm 3.24 & \end{array}$

Note: Level of significance $=p<0.05$

Table 2 RMS EMG activity of masseter muscles at MVC $(\mu \mathrm{V})$ for two groups i.e., the control group (non-TMJD) and the test group (TMJD)

\begin{tabular}{|c|c|c|c|}
\hline \multirow{2}{*}{ Masticatory muscles } & \multicolumn{2}{|c|}{ RMS amplitude value (mean \pm SD, $\mu \mathrm{V}$ ) } & \multirow{2}{*}{$p$} \\
\hline & non-TMJD group & TMJD group & \\
\hline Left masseter muscle & $113.88 \pm 53.44$ & $105.39 \pm 48.40$ & 0.700 \\
\hline Right masseter muscle & $107.92 \pm 48.06$ & $102.75 \pm 62.76$ & 0.830 \\
\hline
\end{tabular}

Note: Level of significance $=p<0.05$

Table 3 Result of the Spearman's correlation test of the association between history of TMJD and activity of masseter muscles on EMG

\begin{tabular}{lcc} 
Masticatory muscles & TMJD correlation coefficient $\left(\boldsymbol{r}_{\boldsymbol{s}}\right)$ & $\boldsymbol{p}$ \\
Left masseter muscle & -0.093 & 0.68 \\
Right masseter muscle & -0.150 & 0.50 \\
\hline
\end{tabular}

Note: Level of significance $=p<0.05, r_{s}>0.3$ 


\section{DISCUSSION}

In this research, the EMG method using surface electrodes was chosen because of its noninvasive procedure which more comfortable compared to the other method by using needle. This method was commonly used in previous studies to identify the relationship between facial growth patterns and muscle activity (Alabdullah et al., 2015). Furthermore, it can compare the muscle activity in patients who had orthodontic treatment (Botelho et al., 2009) and in those with TMJD (Strini et al., 2013).

The examination of masticatory muscle activity was performed on the right and left masseter muscles. The stability of masseter muscle activity could be an important factor related to facial height and mandibular size (Nakamura et al., 2013). Other than that, this muscle was chosen because it is located superficially. Thus, placement of surface electrodes would be easier during examination. Previous study examined the masseter muscles by placing electrodes on the muscular belly of both muscles which is similar to the method of this research (Tartaglia et al., 2011). The assessment of participants was performed during MVC because the method was found to show good reproducibility value (Im et al., 2017). Examination during isometric muscle contraction was also done in several studies (Tartaglia et al., 2011; Strini et al., 2013).

In the present study, female predominance was observed. This finding was similar to a previous study by Rodrigues et al. (2015). Women are at higher risk for TMJD than men because of the hormonal profile (Tonin et al., 2020). Whilst based on the participant's age, the test group was older than the control group. Previous study showed that TMJD was commonly observed in elderly and the number of alterations in the TMJ tends to increase with the patient's age (Tonin et al., 2020). Masseter muscle sEMG activity in the present study was expressed in RMS amplitude value. This method was in accordance with the previous studies, of which the RMS value was used to quantify muscle activity waves generated during EMG examination (Lauriti et al., 2014; Berni et al., 2015). RMS amplitude values were obtained by calculating the roots of the average square of amplitude from the signal wave of muscle activity produced during EMG examination (Tartaglia et al., 2008; Fukuda et al., 2010). This calculation was designed to quantify the intensity and duration of EMG signal waves. The RMS value is a parameter often used because it describes the physiological activity in the motor muscle units during contractions (Tartaglia et al., 2008).

The result showed normal distribution and fairly homogenous data. Thus, a statistical data analysis was performed using parametric tests. However, the value of standard deviation was quite high. This was also found in previous studies (Lauriti et al., 2014; Berni et al., 2015). The standard deviation values were quite high due to several factors influencing the recording of EMG signals. These factors are divided into physiological, anatomical and technical. The physiological factors may include the type of muscle fibers, nerve fiber conduction and body temperature. Whilst the anatomical factors include the diameter of the muscle fibers, position (depth) of the muscles against the electrodes and skin thickness. Technical factors were associated with EMG examination procedure, such as timing of both EMG signal recording and processing of data generated from the examination (Fukuda et al., 2010). The timing of examination affected muscle activity where examination performed in the morning was found to be quite ideal to prevent muscle fatigue (Oncins et al., 2014). Nevertheless, the timing of examination in the present study was quite different due to constraints in the subject's schedule and the limited time for using the EMG instrument in the Neurology Clinic. 
The examination of masseter muscle activity during MVC revealed that the test group had insignificantly lower mean of RMS amplitude compared to the control group. This was in accordance with the result of a previous study (Lauriti et al., 2014). Using different muscle activity parameters, Tartaglia et al. (2008) found that patients without TMJD had a significantly higher muscle activity than those with TMJD. This can occur because the masticatory muscles in patients with TMJD symptoms have decreased efficiency and are at risk of muscle fatigue (Tartaglia et al., 2008). An alteration of muscle activity was also found in a study of cleft patients with TMJD symptoms, which was lower at MVC and higher at rest (SzyszkaSommerfeld et al., 2018). When a muscle suffered some dysfunction, the muscle activity will be decreased during isometric contraction and increased at rest (Davoudi et al., 2015).

The correlation between history of TMJD and masseter muscle activity has insignificantly weak negative correlation. According to this negative relationship, history of TMJD could result in lower muscle activity. Previous study showed that activity of masticatory muscles was reduced in relation to the severity of TMJ hypermobility (Davoudi et al. 2015). In line with that, Woźniak et al. (2015) revealed a phenomenon of increased masseter muscle fatigue relative to the severity of TMJD symptoms. Muscle fatigue was likely to cause lower muscle activity in patients with TMJD. The present study had several limitations, which include small sample size. Moreover, the group with TMJD history was not further classified based on the severity of symptoms. Patients with a history of TMJD who completed orthodontic treatment had a lower masseter muscle activity than those without a history of TMJD. However, the results did not significantly differ. Moreover, there was a negative correlation between history of TMJD and masseter muscle activity, and the result was not statistically significant.

\section{CONCLUSION}

The sEMG examination showed that orthodontically treated patients in TMJD group have reduced masseter muscle activity during MVC, compare to the non-TMJD group.

\section{ACKNOWLEDGEMENTS}

The present study was approved and funded by grant from Directorate Research and Community Service of Universitas Indonesia (Ref. No.: PENG-1/UN2.R3.1/ PPM.00/2019) for student's final project and international scientific publication (HIBAH PITTA).

\section{REFERENCES}

Alabdullah $M$, Saltaji $H$, Abou-Hamed $H$, Youssef M (2015). Association between facial growth pattern and facial muscle activity: A prospective cross-sectional study. Int Orthod, 13(2): 181-194. https://doi.org/10.1016/j.ortho.2015.03.011

Al-Saleh MA, Armijo-Olivo S, Flores-Mir C, Thie NM (2012). Electromyography in diagnosing temporomandibular disorders. f Am Dent Assoc, 143(4): 351-362. https:// doi.org/10.14219/jada.archive.2012.0177

Berni KC, Dibai-Filho AV, Pires PF, Rodrigues-Bigaton D (2015). Accuracy of the surface electromyography RMS processing for the diagnosis of myogenous temporomandibular disorder. f Electromyogr Kinesiol, 25(4): 596-602. https://doi.org/10.1016/j.jelekin.2015.05.004

Botelho AL, Melchior Mde O, da Silva AM, da Silva MA (2009). Electromyographic evaluation of neuromuscular coordination of subject after orthodontic intervention. f Cranio, 27(3): 152-158. https://doi.org/10 $.1179 /$ crn.2009.023 
Davoudi A, Haghighat A, Rybalov O, Shadmehr E, Hatami A (2015). Investigating activity of masticatory muscles in patients with hypermobile temporomandibular joints by using EMG. F Clin Exp Dent, 7(2): e310e315. https://doi.org/10.4317/jced.52125

Fukuda TY, Echeimberg JO, Pompeu JE, Garcia Lucareli PR, Garbelotti S, Gimenes RO et al. (2010). Root mean square value of the electromyographic signal in the isometric torque of the quadriceps, hamstrings and brachial biceps muscles in female subjects. f Appl Res, 10(1): 32-39.

Henrikson T, Nilner M (2003). Temporomandibular disorders, occlusion and orthodontic treatment. F Orthod, 30(2): 129-137. https://doi.org/10.1093/ortho/30 .2 .129

Im YG, Han SH, Park JI, Lim HS, Kim BG, Kim JH (2017). Repeatability of measurements of surface electromyographic variables during maximum voluntary contraction of temporalisis and masseter muscles in normal adults. F Oral Sci, 59(2): 233-245. https://doi.org/10.2334/josnusd.16-0434

Lauriti L, Motta LJ, de Godoy CH, BiasottoGonzalez DA, Politti F, MesquitaFerrari RA et al. (2014). Influence of temporomandibular disorder on temporalis and masseter muscles and occlusal contacts in adolescents: An electromyographic study. BMC Musculoskelet Disord, 15: 123. https://doi.org/10.1186/1471-2474-15-123

Masci C, Ciarrocchi I, Spadaro A, Necozione S, Marci MC, Monaco A (2013). Does orthodontic treatment provide a real functional improvement? A case control study. BMC Oral Health, 13: 57. https://doi.org/10.1186/1472-6831-13-57

Nakamura K, Hara A, Nakata S, Hyakutake H, Takahashi I (2013). Relationship between the stability of muscle activity in the masseter muscle and craniofacial morphology. Orthod Waves, 72(2): 55-62. https://doi.org/10.1016/j.odw.2013.01.021
Oncins MC, Vieira MM, Bommarito S (2014). Electromyography of the masticatory muscles: Analysis in the original and RMS value. Rev CEFAC, 16(4): 1215-1220.

Politti F, Casellato C, Kalytczak MM, Garcia MB, Biasotto-Gonzalez DA (2016). Characteristics of EMG frequency bands in temporomandibullar disorders patients. f Electromyogr Kinesiol, 31: 119-125. https://doi.org/10.1016/j.jelekin.2016.10 .006

Rodrigues CA, de Oliveira Melchior M, Magri LV, Mestriner W Jr, Mazzetto MO (2015). Is the masticatory function changed in patients with temporomandibular disorder? Braz Dent J, 26(2): 181-185. https://doi .org/10.1590/0103-6440201300198

Strini PJ, Strini PJ, Barbosa Tde S, Gavião MB (2013). Assessment of thickness and function of masticatory and cervical muscles in adults with and without temporomandibular disorders. Arch Oral Biol, 58(9): 1100-1108. https://doi.org/10 $.1016 /$ j.archoralbio.2013.04.006

Sumonsiri P, Thongudomporn U (2017). Surface electromyographic studies on masticatory muscle activity related to orthodontics: a review of literature. I Dent Assoc Thai, 67(2): 107-118.

Szyszka-Sommerfeld L, Matthews-Brzozowska T, Kawala B, Mikulewicz M, Machoy M, Więckiewicz W et al. (2018). Electromyographic analysis of masticatory muscles in cleft lip and palate children with pain-related temporomandibular disorders. Pain Res Manag, 2018: 4182843. https://doi.org/10.1155/2018/4182843

Tartaglia GM, Lodetti G, Paiva G, De Felicio CM, Sforza C (2011). Surface electromyographic assessment of patients with long lasting temporomandibular joint disorder pain. F Electromyogr Kinesiol, 21(4): 659-664. https://doi.org/10.1016/ j.jelekin.2011.03.003 
Tartaglia GM, Moreira Rodrigues da Silva MA, Bottini S, Sforza C, Ferrario VF (2008). Masticatory muscle activity during maximum voluntary clench in different research diagnostic criteria for temporomandibular disorders Masticatory muscle activity during maximum voluntary clench in different research diagnostic criteria for temporomandibul. Man Ther, 13(5): 434-440. https://doi.org/10.1016/j .math.2007.05.011

Tonin RH, Iwaki Filho L, Grossmann E, Lazarin RO, Pinto GNS, Previdelli ITS et al. (2020). Correlation between age, gender, and the number of diagnoses of temporomandibular disorders through magnetic resonance imaging: A retrospective observational study. Cranio, 38(1): 34-42. https://doi.org/10.1080/0886 9634.2018 .1476078
Woźniak K, Lipski M, Lichota D, SzyszkaSommerfeld L (2015). Muscle fatigue in the temporal and masseter muscles in patients with temporomandibular dysfunction. BioMed Res Int, 2015: 269734. https://doi.org/10.1155/2015/269734 